Proceedings

\title{
Polydimethylsiloxane (PDMS) Waveguide Sensor Detecting Fluid Flow Velocity by Mimicking the Fish Lateral Line Organ ${ }^{\dagger}$
}

\author{
Bianca Wiesmayr ${ }^{1, *}$, Michael Krieger ${ }^{2}$, Werner Baumgartner ${ }^{1}$ and Anna T. Stadler ${ }^{1}$ \\ 1 Institute of Biomedical Mechatronics, Johannes Kepler University Linz, Linz 4040, Austria; \\ werner.baumgartner@jku.at (W.B.); anna.stadler@jku.at (A.T.S.) \\ 2 Institute of Fluid Mechanics and Heat Transfer, Johannes Kepler University Linz, \\ Linz 4040, Austria; michael.krieger@jku.at \\ * Correspondence: bianca.wiesmayr@jku.at; Tel.: +43-732-2468-4809 \\ + Presented at the Eurosensors 2018 Conference, Graz, Austria, 9-12 September 2018. \\ Published: 23 November 2018
}

\begin{abstract}
Accurate measurement of fluid flow velocities is challenging but essential in many disciplines. Inspiration of possible measurement methods can come from nature, for example from the lateral line organ of fish, which is comprised of hair cells embedded in a gelatinous cupula. When the cupula is deflected by water movement, the hair cells initiate neural signals that generate an accurate image of the fish's surroundings. We built a flow sensor mimicking a hair cell, yet coupled it with an optical detection method. Fluid flow bends the waveguide; this leads to a measurable light loss that depends linearly on the waveguide deflection.
\end{abstract}

Keywords: biomimetic flow sensor; PDMS waveguide; optical detection principle

\section{Introduction}

In biomimetics, natural structures are analyzed to develop novel technologies and optimize existing ones. A model for flow sensing, for example, can be found in the lateral line of fish. This organ consists of hundreds of neuromasts spread all across the head and body of the fish. The lateral line provides survival information such as location of prey or predators and enables intraspecific communication and schooling. The structure of the lateral line is based on neuromasts [1].

A neuromast is comprised of hair cells embedded in a gelatinous cupula. Fluid flow deflects the cupula and thus bends the hair, which results in a neural signal. The neuromasts in the lateral line can be classified into two types, the superficial (SN) and canal neuromasts $(\mathrm{CN})$. While SNs occur on the surface of the skin, CNs are situated in a canal under the skin, between two pores of this canal. SNs measure the fluid flow velocity of the bulk water flow, and CNs record pressure differences between the canal pores [2].

Numerous artificial flow sensors mimicking neuromasts have been developed. The biomimetic sensors created so far are based on hot-wire anemometry [3], capacity [4], piezoelectricity [5], and ionic polymer-metal composite [6]. Furthermore, a polydimethylsiloxane (PDMS) sensor was presented $[7,8]$, which is based on optical detection and implemented as a micro-electromechanical system (MEMS).

In several studies [3,5,6,9-11], biomimetic flow sensors were combined to an artificial lateral line. An artificial lateral line may be utile in autonomous underwater navigation [12]. Additionally, the individual sensors were used for gas flow sensing [7], underwater flow sensing [4-8], and tap water measurements [8]. 
The purpose of this study was to investigate a novel, low-cost and robust fluid flow sensor. We recently presented a fluid mechanical description of the sensor [13]. The design of our sensory system also allows for flow measurements in opaque and erosive fluids, a possible limitation of the measurement set-up described by a German research group $[7,8]$.

\section{Materials and Methods}

The hair cell of the lateral line is mimicked by an elastic PDMS waveguide that is exposed to fluid flow. The optical detection represents the neural signals generated in the neuromast. Red light is coupled into the waveguide by a LED (XP-E2, Cree) and focused by a lens (18 ${ }^{\circ}$, Carclo Optics). At the other end of the waveguide, the remaining light is detected by a photodiode (BPX61, Osram). The operation principle is shown in Figure 1a. As the waveguide is directly connected to the LED and the photodiode, measurements are feasible in opaque environments.

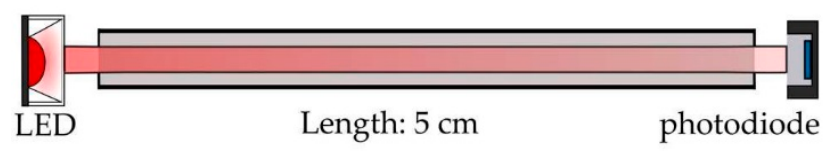

(a)

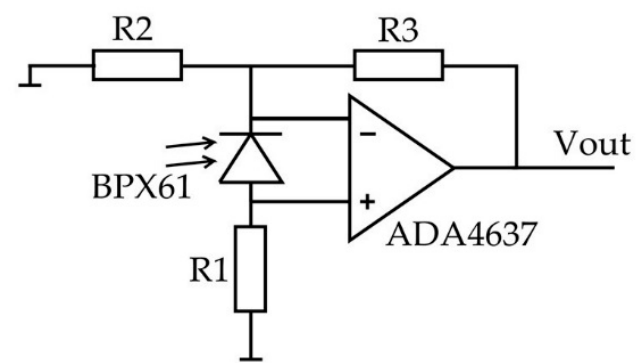

(b)

Figure 1. Measuring the deflection of the waveguide. (a) Schematic operation concept of the optical sensory system that is comprised of a LED, an optical waveguide and a photodiode [14]. (b) Photodiode amplifier.

\subsection{PDMS Waveguide}

The fabrication process of the optical waveguide was described and illustrated by Stadler et al. [13]. The core is fabricated of the elastomer Sylgard 184, which has a slightly higher refractive index than the cladding material RTV 615. An additional sealing layer (RTV 3145) protects the waveguide against erosion and absorbs light to increase the light loss. A small refractive index contrast results in high bending sensitivity of the waveguide [14]. Schneider et al. analyzed the optical properties of PDMS [15], showing that Sylgard 184 has an absorption minimum around $620 \mathrm{~nm}$. Therefore, red light was chosen for the optical path.

\subsection{Photodiode and Photodiode Amplifier}

The photodiode has a high sensitivity of $0.7 \mathrm{~A} / \mathrm{W}$, allowing the detection of low light levels. Although the device has a large active area of $7 \mathrm{~mm}^{2}$, the capacitance amounts to only $72 \mathrm{pF}$ [16]. The signal is amplified by a photodiode amplifier; the output voltage therefore depends linearly on the photocurrent. As proposed by Horowitz and Hill [17], the photodiode is placed between the inputs of the opamp (ADA4637-1ARZ, Analog Devices, Boston, MA, US) for zero bias voltage and noise reduction. The design was optimized for low noise and low temperature dependency. Resistor R1 (see Figure $1 \mathrm{~b}$ ) converts the photocurrent into a voltage and was chosen to $400 \mathrm{k} \Omega$. The resistors R2 and $\mathrm{R} 3$ of 1 and $60 \mathrm{k} \Omega$, respectively, define the amplification. The circuitry was fabricated as a printed circuit board (PCB) with high-precision surface mount devices (SMD). The photodiode (through-hole technology) was placed directly on the PCB. For increased stability, a capacitor (70 pF) was added parallel to R4. Decoupling capacitors $(100 \mathrm{nF})$ are placed close to the opamp's supply pins.

\subsection{Measurements}

The LED is driven by a constant current source of $350 \mathrm{~mA}$ and the opamp has a symmetric supply of $15 \mathrm{~V}$. The output voltage was recorded using a data acquisition device (DAQ, NI USB-6228, 
National Instruments) connected to a laptop via USB. A LabVIEW script (LabVIEW 2014, National Instruments) evaluated and saved the data. The PDMS waveguide was tested for masses bending the sensor to simulate deflection of the waveguide in fluids. For each measurement, the reference voltage $\mathrm{V} 0$ of the unbent waveguide was recorded. The output voltage decreased when a mass was attached to stress the waveguide. Six cycles with 50 measurements each were conducted. The masses were implemented as lead-filled micro test tubes between 0.1 and $5 \mathrm{~g}$. Data was recorded for $5 \mathrm{~s}$ with $\mathrm{f}=4$ $\mathrm{kHz}$ and the mean value was calculated.

\section{Results}

The measurement results are illustrated in Figure 2 as light loss in percent of the reference voltage V0 over the attached masses. The results demonstrate that the light loss is linearly dependent on the induced bending. At a mass of $5 \mathrm{~g}$, the mean light loss is $46.7 \%$; therefore, the average loss for $\Delta \mathrm{m}=0.1 \mathrm{~g}$ amounts to approximately $1 \%$. The standard deviation increases with the attached mass.

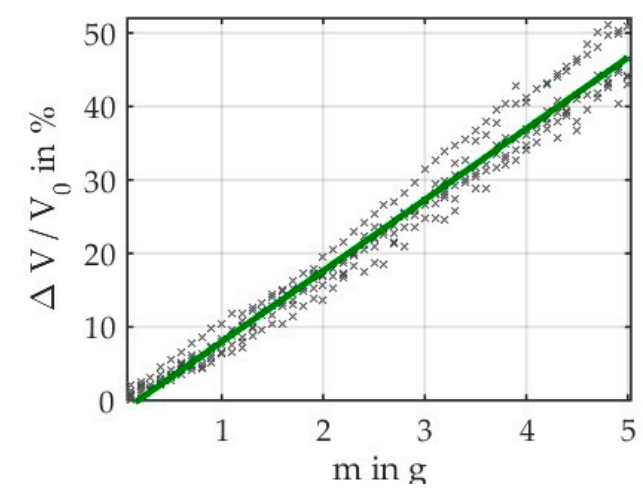

(a)

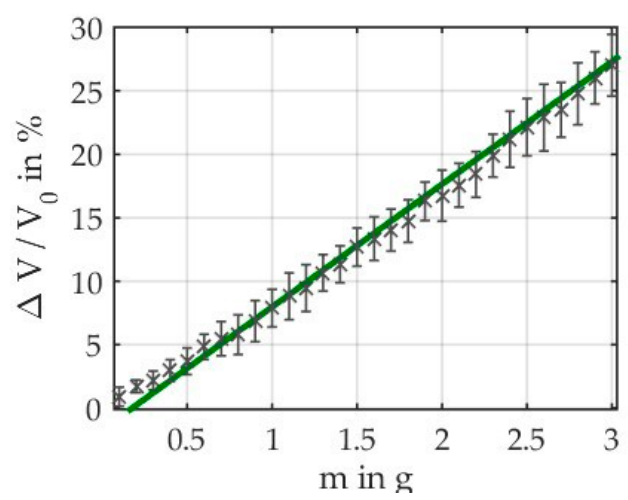

(b)

Figure 2. Measurement results showing that light loss depends linearly on the bending mass. (a) Results of six measurements with masses from 0 to $5 \mathrm{~g}$ and step size $0.1 \mathrm{~g}$ are illustrated including linear approximation. (b) For the bending masses from 0 to $3 \mathrm{~g}$, the average measurement result including error bars is shown.

\section{Discussion}

The standard deviation is below $2.5 \%$ for more than half of the data. In these cases, the bending mass may be identified with an accuracy $\pm 0.25 \mathrm{~g}$. The standard deviation increases for higher masses (ranging from $0.75 \%$ at $0.1 \mathrm{~g}$ to $4.36 \%$ at $4.9 \mathrm{~g}$ ), because the attached masses twist the fiber. This error is inherent to the measurement setup and therefore, it is expected to be lower in continuous air flow.

\section{Conclusions}

The purpose of this study was to investigate a novel, low-cost and robust flow sensory system for fluid flow sensing. A PDMS fiber was used as an optical waveguide with high sensitivity for bending losses. We demonstrated, by straining the waveguide with masses from 0 to $5 \mathrm{~g}$ that the light loss is linearly dependent on the induced bending. This robust flow sensor based on the biomimetic principle of the lateral line can measure the flow velocity of opaque or erosive fluids. The next steps in sensor development should include testing the device in liquid flow. As the sensor measures bulk flow velocity of fluids, it can be considered as biomimetic $\mathrm{SN}$ sensor.

Acknowledgments: The authors wish to thank the Center for Surface and Nanoanalytics at Johannes Kepler University Linz, Austria (ZONA). Robert Leimlehner (ZONA and Institute of Experimental Physics) and Philip Lindner (ZONA and Institute of Semiconductor and Solid State Physics) have contributed greatly by designing and fabricating the waveguide clamps for the measurement system. 
Conflicts of Interest: The authors declare no conflict of interest. The founding sponsors had no role in the design of the study; in the collection, analyses, or interpretation of data; in the writing of the manuscript, and in the decision to publish the results.

\section{References}

1. Bleckmann, H.; Zelick, R. Lateral line system of fish. Integr. Zool. 2009, 4, 13-25.

2. Coombs, S.; Montgomery, J.C. The Enigmatic Lateral Line System. In Comparative Hearing: Fish and Amphibians; Fay, R.R., Popper, A.N., Eds.; Springer: New York, NY, USA, 1999; pp. 319-362.

3. Yang, Y.; Chen, J.; Engel, J.; Pandya, S.; Chen, N.; Tucker, C.; Coombs, S.; Jones, D.L.; Liu, C. Distant touch hydrodynamic imaging with an artificial lateral line. Proc. Natl. Acad. Sci. USA 2006, 103, 18891-18895.

4. Izadi, N.; de Boer, M.J.; Berenschot, J.W.; Krijnen, G.J.M. Fabrication of superficial neuromast inspired capacitive flow sensors. J. Micromech. Microeng.2010, 20, 085041.

5. Asadnia, M.; Kottapalli, A.G.P.; Miao, J.; Warkiani, M.E.; Triantafyllou, M.S. Artificial fish skin of selfpowered micro-electromechanical systems hair cells for sensing hydrodynamic flow phenomena. J. R. Soc. Interface 2015, 12, 20150322.

6. Abdulsadda, A.T.; Tan, X. An artificial lateral line system using IPMC sensor arrays. Int. J. Smart Nano Mater. 2012, 3, 226-242.

7. Herzog, H.; Klein, A.; Bleckmann, H.; Holik, P.; Schmitz, S.; Siebke, G.; Tätzner, S.; Lacher, M.; Steltenkamp, S. Micro-biomimetic flow sensors: Introducing light-guiding PDMS structures into MEMS. Bioinspir. Biomim. 2015, 10, 36001.

8. Herzog, H.; Steltenkamp, S.; Klein, A.; Tätzner, S.; Schulze, E.; Bleckmann, H. Micro-Machined Flow Sensors Mimicking Lateral Line Canal Neuromasts. Micromachines 2015, 6, 1189-1212.

9. Yang, Y.; Klein, A.; Bleckmann, H.; Liu, C. Artificial lateral line canal for hydrodynamic detection. Appl. Phys. Lett. 2011, 99, 23701.

10. Klein, A.T.; Kaldenbach, F.; Rüter, A.; Bleckmann, H. What We Can Learn from Artificial Lateral Line Sensor Arrays. Adv. Exp. Med. Biol. 2016, 875, 539-545.

11. Chen, J.; Engel, J.; Chen, N.; Pandya, S.; Cocombs, S.; Liu, C. Artificial Lateral Line and Hydrodynamic Object Tracing. In Micro Electro Mechanical Systems; IEEE: Istanbul, Turkey, 2006.

12. Nguyen, N.; Jones, D.L.; Yang, Y.; Liu, C. Flow Vision for Autonomous Underwater Vehicles via an Artificial Lateral Line. EURASIP J. Adv. Signal Process. 2011, 2011, 51.

13. Stadler, A.T.; Wiesmayr, B.; Krieger, M.; Baumgartner, W. An optical sensory principle for spirometry. In Sensors and Measuring Systems; VDE: Nuremberg, Germany, 2018.

14. Snyder, A.W.; Love, J.D. Optical Waveguide Theory; Springer: Boston, MA, USA, 1983.

15. Schneider, F.; Draheim, J.; Kamberger, R.; Wallrabe, U. Process and material properties of polydimethylsiloxane (PDMS) for Optical MEMS. Sens. Actuators A Phys. 2009, 151, 95-99.

16. Osram Opto Semiconductors. Silicon PIN Photodiode BPX61, 1st ed.; Osram Opto Semiconductors GmbH: Regensburg, Germany, 2015.

17. Horowitz, P.; Hill, W. The Art of Electronics, 11th ed.; Cambridge University Press: Cambridge, UK, 2017.

(C) 2018 by the authors. Licensee MDPI, Basel, Switzerland. This article is an open access article distributed under the terms and conditions of the Creative Commons Attribution (CC BY) license (http://creativecommons.org/licenses/by/4.0/). 